\title{
GOODS-Herschel: the impact of galaxy-galaxy interactions on the far-infrared properties of galaxies
}

\author{
H. S. Hwang ${ }^{1}$, D. Elbaz ${ }^{1}$, M. Dickinson ${ }^{2}$, V. Charmandaris ${ }^{3,4, \star}$, E. Daddi ${ }^{1}$, D. Le Borgne ${ }^{6}$, V. Buat ${ }^{5}$, G. E. Magdis ${ }^{1,7}$, \\ B. Altieri ${ }^{8}$, H. Aussel ${ }^{1}$, D. Coia $^{8}$, H. Dannerbauer ${ }^{1}$, K. Dasyra ${ }^{1}$, J. Kartaltepe ${ }^{2}$, R. Leiton ${ }^{1}$, B. Magnelli ${ }^{9}$, \\ P. Popesso ${ }^{9}$, and I. Valtchanov ${ }^{8}$
}

\author{
${ }^{1}$ Laboratoire AIM-Paris-Saclay, CEA/DSM/Irfu, CNRS, Université Paris Diderot, CE-Saclay, 91191 Gif-sur-Yvette, France \\ e-mail: hwang.ho.seong@gmail.com \\ 2 National Optical Astronomy Observatory, 950 North Cherry Avenue, Tucson, AZ 85719, USA \\ 3 Department of Physics and Institute of Theoretical \& Computational Physics, University of Crete, 71003 Heraklion, Greece \\ ${ }^{4}$ IESL/Foundation for Research and Technology - Hellas, 71110 Heraklion, Greece \\ 5 Laboratoire d'Astrophysique de Marseille, OAMP, Université Aix-Marseille, CNRS, 38 rue Frédéric Joliot-Curie, \\ 13388 Marseille Cedex 13, France \\ ${ }^{6}$ Institut d'Astrophysique de Paris, UMR 7095, CNRS, UPMC Univ. Paris 06, 98bis boulevard Arago, 75014 Paris, France \\ 7 Department of Physics, University of Oxford, Keble Road, Oxford OX1 3RH, UK \\ 8 Herschel Science Centre, European Space Astronomy Centre, Villanueva de la Cañada, 28691 Madrid, Spain \\ 9 Max-Planck-Institut für Extraterrestrische Physik (MPE), Postfach 1312, 85741, Garching, Germany
}

Received 14 June 2011 / Accepted 8 September 2011

\section{ABSTRACT}

\begin{abstract}
Aims. We study the impact of galaxy-galaxy interactions on the far-infrared properties of galaxies and its evolution at $0<z<1.2$. Methods. Using the high- $z$ galaxies in the fields of Great Observatories Origins Deep Survey (GOODS) observed by the Herschel Space Observatory in the framework of the GOODS-Herschel key program and the local IRAS or AKARI-selected galaxies in the field of Sloan Digital Sky Survey Data Release 7, we investigate the dependence of galaxy properties on the morphology of and the distance to the nearest neighbor galaxy.

Results. We find that the star-formation rates (SFRs) and the specific SFRs (SSFRs) of galaxies on average depend on the morphology of and the distance to the nearest neighbor galaxy in this redshift range. When a late-type galaxy has a close neighbor galaxy, the SFR and the SSFR increase as it approaches a late-type neighbor, which is supported by Kolmogorov-Smirnov (K-S) and Monte Carlo (MC) tests with a significance level of $>99 \%$. However, the SFR and the SSFR decrease or do not change much as the galaxy approaches an early-type neighbor. The bifurcations of SFRs and SSFRs depending on the neighbor's morphology seem to occur at $R_{n} \approx 0.5 r_{\text {virnei }}$ (virial radius of the neighbor), which is supported by K-S and MC tests with a significance level of $>98 \%$. For all redshift bins, the SSFRs of late-type galaxies interacting with late-type neighbors are increased by factors of about $1.8 \pm 0.7$ and $4.0 \pm 1.2$ compared to those of non-interacting galaxies when the pair separation is smaller than $0.5 r_{\text {virnei }}$ and $0.1 r_{\text {vir,nei }}$, respectively. The dust temperature of both local and high- $z$ late-type galaxies that strongly interact with late-type neighbors (i.e. $R_{\mathrm{n}} \leq 0.1 r_{\text {vir,nei }}$ ) appears to be higher than that of non-interacting galaxies with a significance level of 96-99\%. However, the dust temperature of local late-type galaxies that strongly interact with early-type neighbors seems to be lower than or similar to that of non-interacting galaxies.

Conclusions. Our results suggest that galaxy-galaxy interactions and mergers have been strongly affecting the SFR and the dust properties of star-forming galaxies over at least 8 billion years.
\end{abstract}

Key words. galaxies: active - infrared: galaxies - galaxies: evolution - galaxies: interactions - galaxies: formation galaxies: starburst

\section{Introduction}

In the hierarchical picture of galaxy formation, galaxies are formed and grow through continuous interactions and mergers with other galaxies. These galaxy-galaxy interactions and mergers are expected to strongly affect galaxy properties such as morphology, luminosity, structure parameters, star-formation rate (SFR), or dust properties (see Struck 2006 for a review).

Since Toomre (1977) first suggested that the merger between spiral galaxies can form elliptical galaxies, there is growing evidence for a change in galaxy morphology (e.g., Park et al. 2008) and galaxy structure with merger (e.g., Nikolic et al. 2004; Patton et al. 2005; Hernández-Toledo et al. 2005; Park \& Choi 2009). For example, Park et al. (2008) showed that galaxy

\footnotetext{
* Chercheur Associé, Observatoire de Paris, 75014 Paris, France.
}

morphology and luminosity strongly depend on the distance to and the morphology of the nearest neighbor galaxy. When a galaxy is located within the virial radius of its nearest neighbor, its morphology tends to be the same as that of the neighbor. This indicates an important role of hydrodynamical interactions with neighbors within the virial radius. This morphological conformity was also found between hosts and their satellite galaxies (Ann et al. 2008; Wang et al. 2010b), and between galaxies in galaxy clusters (Park \& Hwang 2009).

For the star-formation activity (SFA), Larson \& Tinsley (1978) first noted that morphologically normal and peculiar galaxies show very different optical color distributions, which suggests that the SFA of peculiar galaxies is enhanced compared to those of normal galaxies. Many studies have extensively investigated this enhancement of SFA in paired galaxies 
(e.g., Condon et al. 1982; Keel et al. 1985; Kennicutt et al. 1987; Barton et al. 2000; Lambas et al. 2003; Nikolic et al. 2004; Alonso et al. 2004; De Propris et al. 2005; Geller et al. 2006; Woods \& Geller 2007; Li et al. 2008; Ellison et al. 2008; Knapen \& James 2009; Perez et al. 2009; Park \& Choi 2009; Hwang et al. 2010a; Xu et al. 2010; Darg et al. 2010; Patton et al. 2011). In summary, the SFA of galaxies seems to strongly depend on the morphology and the mutual separation of galaxies in pairs. For example, the SFR of a galaxy is increased when the nearest neighbor has a late morphological type, but is decreased or remains the same when the neighbor is an early-type galaxy (Park \& Choi 2009; Hwang et al. 2010a; Xu et al. 2010). There are two characteristic pair-separation scales where the SFA abruptly changes: the virial radius ( $r_{\text {vir,nei }}$, to be defined in Sect. 2.4) of the nearest neighbor galaxy where the effects of galaxy interaction emerge (roughly a few hundred kpc for bright galaxies) and $\sim 0.05 r_{\text {vir,nei }}$ where the galaxies in pairs start to merge (Park $\&$ Choi 2009). However, some other studies found no significant difference in galaxy properties between isolated and paired galaxies (e.g., Yee \& Ellingson 1995; Patton et al. 1997; Bergvall et al. 2003). For high- $z$ galaxies, the evolution of the fraction of interacting galaxies has been studied extensively and is found to increase with redshift (e.g., Zepf \& Koo 1989; Kartaltepe et al. 2007; Bridge et al. 2010), but there are few studies focusing on the enhancement of SFA in paired galaxies (e.g., Lin et al. 2007; de Ravel et al. 2009; Woods et al. 2010; Wong et al. 2011).

For dust-enshrouded systems such as luminous infrared galaxies (LIRGs), one might have expected that not only the SFR but also the dust properties such as dust temperature or dust mass change as a function of pair separation. Interestingly, Telesco et al. (1988) found the highest dust color temperature (i.e., the flux density ratio between IRAS 60 and $100 \mu \mathrm{m}$ ) for galaxies in pairs with the smallest separation. On the other hand, Lutz et al. (1998) and Rigopoulou et al. (1999) did not find any correlation between the line-to-continuum ratio for the $7.7 \mu \mathrm{m}$ PAH emission feature (i.e. SF vs. AGN activity) and the nuclear separation of the interacting components of ultraluminous infrared galaxies (ULIRGs). Klaas et al. (2001) also searched for the differences in the mid-infrared (MIR), far-infrared (FIR) and submm luminosity ratios of interacting and non-interacting ULIRGs, but did not find any noticeable difference depending on the merging stage. However, Xilouris et al. (2004) found evidence for an increase in the 100 - to $850-\mu \mathrm{m}$ flux density ratio (a proxy for the mass fraction of the warm and cold dust) with the merging sequence. They argue that the contradictory results may be caused by the different sample selection. Klaas et al. (2001) did not order their interacting ULIRG systems on the basis of the pair separation. Their samples span a small range in bolometric luminosity, therefore, they might have missed some interacting systems in the very early stage of merging. It is also noted that Smith et al. (2007) found no significant change in Spitzer MIR colors with pair separation in their sample of tidally distorted premerger interacting galaxy pairs. This may be because their sample was selected to be in the early stage of interactions.

Thanks to the advent of the Herschel Space Observatory (Pilbratt et al. 2010) with its very wide wavelength coverage (70-500 $\mu \mathrm{m})$, we are now able to have both the "Wien" and "Rayleigh-Jeans" sides of the FIR spectral energy distributions (SEDs) of high- $z$ galaxies, and to have robust SFR and dust temperature measurements for them (e.g., Elbaz et al. 2010, 2011; Hwang et al. 2010b; Magdis et al. 2010a,b). In this paper, we investigate the impact of galaxy-galaxy interactions on galaxy properties, focusing on FIR properties of high- $z$ galaxies at $0.4 \lesssim z \lesssim 1.2$ by taking advantage of the wide wavelength coverage of the Photodetector Array Camera and Spectrometer (PACS; Poglitsch et al. 2010) and Spectral and Photometric Imaging REceiver (SPIRE; Griffin et al. 2010) instruments onboard Herschel in the fields of Great Observatories Origins Deep Survey (GOODS; Dickinson et al. 2003; Giavalisco et al. 2004). To compare these results with those of local galaxies, we also use a sample of galaxies at $z<0.1$ that were covered by IRAS and AKARI satellites (Murakami et al. 2007) in the field of Sloan Digital Sky Survey (SDSS, York et al. 2000). The IRAS and AKARI all-sky survey data contain flux density measurements at $12-160 \mu \mathrm{m}$, which can probe both the "Wien" and "RayleighJeans" sides of the peak of IR SEDs of local galaxies in a way similar to that of Herschel for high- $z$ galaxies. Section 2 describes the data used in this study, and the dependence of galaxy properties on the distance to and the morphology of the nearest neighbor galaxies is given in Sect. 3. Discussion and conclusions are given in Sects. 4 and 5, respectively. Throughout, we adopt $h=0.7$ and a flat $\Lambda$ CDM cosmology with density parameters $\Omega_{\Lambda, 0}=0.73$ and $\Omega_{\mathrm{m}, 0}=0.27$.

\section{Data}

\subsection{GOODS sample}

We used a spectroscopic sample of galaxies in GOODS, which is a deep multiwavelength survey covering two carefully selected regions including the Hubble Deep Field North (HDF-N) and the Chandra Deep Field South (CDF-S). Hereafter, the two GOODS fields centered on HDF-N and CDF-S are called GOODS-N and GOODS-S, respectively. The combined area of the two fields is approximately $320 \mathrm{arcmin}^{2}$.

The GOODS fields were observed by Herschel in the GOODS-Herschel key program (Elbaz et al. 2011). The full $10^{\prime} \times 16^{\prime}$ GOODS-N field was observed with both PACS $(100$ and $160 \mu \mathrm{m})$ and $\operatorname{SPIRE}(250,350$, and $500 \mu \mathrm{m})$. A smaller region within the GOODS-S field $\left(\approx 10^{\prime} \times 10^{\prime}\right)$ was observed with PACS only. We supplemented these data with public SPIRE images covering the full $10^{\prime} \times 16^{\prime}$ GOODS-S field, which was originally taken in the HerMES key program (Oliver et al., in prep.). Source extraction on these PACS and SPIRE images was performed at the prior positions of Spitzer $24 \mu \mathrm{m}$-selected sources, and details are described in Elbaz et al. (2011). Note that this extraction method with $24 \mu \mathrm{m}$-selected sources recovers more than $99 \%$ of Herschel sources (Magdis et al. 2011). We used flux densities in PACS bands down to $3 \sigma$ limits of 1.1 and $2.7 \mathrm{mJy}(0.8$ and $2.4 \mathrm{mJy})$ at 100 and $160 \mu \mathrm{m}$ in GOODS-N (GOODS-S), respectively. SPIRE measurements are used down to $5 \sigma$ limits of $5.7,7.2$, and $9.0 \mathrm{mJy}$ at 250,350 and $500 \mu \mathrm{m}$, respectively (see Table 1 in Elbaz et al. 2011 for more details about the noise properties). By combining Herschel data with the existing multi-wavelength data, we made a band-merged catalog of GOODS galaxies using the photometric data at HST ACS BViz, CFHT/WIRCam JK (North; Wang et al. 2010a) and VLT/ISAAC JHK (South; Retzlaff et al. 2010), Spitzer IRAC 3.6, 4.5, 5.8, $8 \mu \mathrm{m}$ and MIPS 24 and $70 \mu \mathrm{m}$, Herschel PACS 100 and $160 \mu \mathrm{m}$, and Herschel SPIRE 250, 350 and $500 \mu \mathrm{m}$.

Among the sources in the band-merged catalog, we used 3630 and 3181 galaxies whose spectroscopic redshifts are reliable over the whole fields of GOODS-N (Cohen et al. 2000; Cowie et al. 2004; Wirth et al. 2004; Reddy et al. 2006; Barger et al. 2008; Cooper et al. 2011; Stern et al., in prep.) and GOODS-S (Szokoly et al. 2004; Le Fèvre et al. 2004; Mignoli et al. 2005; Vanzella et al. 2005, 2006, 2008; Ravikumar et al. 2007; Popesso et al. 2009; Kurk et al. 2009; Balestra et al. 2010; 


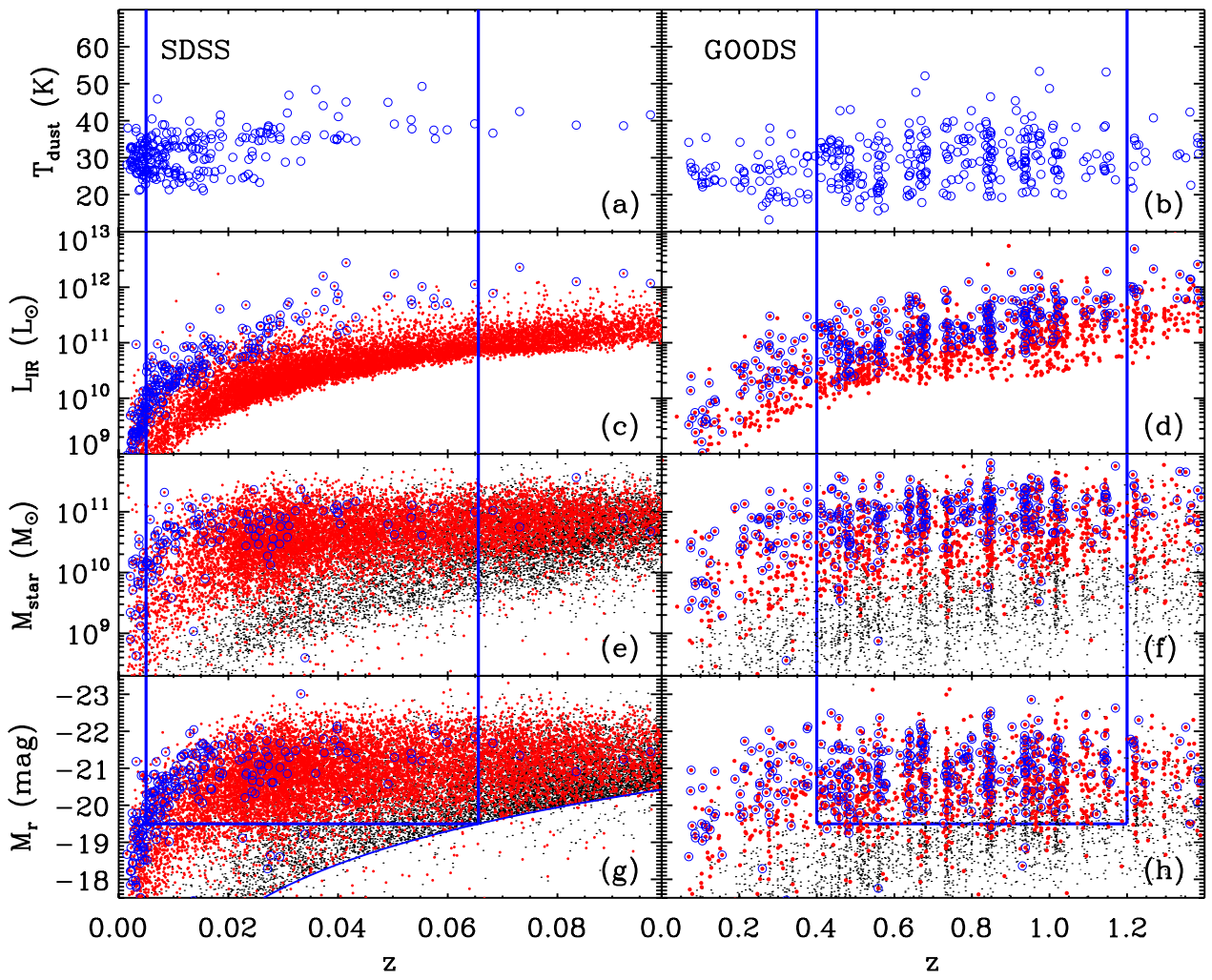

Fig. 1. (a)-b)) Dust temperature ( $\left.T_{\text {dust }}\right)$, (c)-d)) IR luminosity, (e)-f)) stellar mass, and (g)-h)) evolution-corrected, rest frame $r$-band absolute magnitude vs. spectroscopic redshift for galaxies in (left) SDSS and (right) GOODS. Red dots indicate FIR-selected galaxies. Black dots denote galaxies without FIR detection in the spectroscopic sample of galaxies (only $3 \%$ of SDSS galaxies in the total sample are shown). Open blue circles denote galaxies with $T_{\text {dust }}$ measurements. Blue solid lines in $\left.(\mathbf{g})-\mathbf{h}\right)$ ) define the volume-limited samples of SDSS galaxies at $0.005 \leq z \leq 0.0656$ and of GOODS galaxies at $0.4 \leq z \leq 1.2$, respectively. The bottom curve in $(\mathrm{g})$ indicates the apparent magnitude limit $\left(m_{r}=17.77\right)$ for the main galaxy sample in SDSS using the mean $K$-correction relation given by Eq. (2) of Choi et al. (2007).

Silverman et al. 2010; Xia et al. 2011), respectively. The rest frame $r$-band absolute magnitude $M_{r}$ of galaxies was computed based on the ACS plus near-infrared (NIR) photometry with $K$-corrections (Blanton \& Roweis 2007). It was computed in fixed bandpasses, shifted to $z=0.1$ to be compared with local SDSS galaxies. The 1.1( $z-0.1)$ term was added to $M_{r}$ for the evolution correction (Wolf et al. 2003). We calculated stellar masses using the photometric data upto IRAC $4.5 \mu \mathrm{m}$ with a Salpeter IMF (Salpeter 1955). We used the code Z-PEG with a galaxy evolution model PÉGASE.2 (Fioc \& Rocca-Volmerange 1999; Le Borgne \& Rocca-Volmerange 2002; see Elbaz et al. 2011 for details).

We adopted the galaxy morphology information [early types (E/S0) and late types (S/Irr)] from Hwang \& Park (2009) that is based on the visual inspection of ACS BViz images. We performed additional visual classification for the galaxies in the volume-limited sample of galaxies shown in the right panels of Fig. 1 that are not included in Hwang \& Park (2009).

We computed the IR luminosity $\left(L_{\mathrm{IR}}\right)$ for 903 and 828 galaxies with spectroscopic redshifts in GOODS-N and -S, respectively, detected in at least one out of the five PACS/SPIRE bands. We fitted the flux densities at $\lambda_{\text {rest }} \geq 30 \mu \mathrm{m}$ by allowing the normalization of the SED templates of Chary \& Elbaz (2001, CE01) to vary and choosing the one that minimizes the $\chi^{2}$ values. When there were two or less data points to fit (i.e. $N \leq 2$ ), we fitted the flux densities without allowing the normalization of the templates (i.e. standard CE01 technique). There were some cases with only one FIR band used for $L_{\mathrm{IR}}$ measurement, but the IR luminosities extrapolated from a single passband were found to agree very well with those measured with all FIR bands with an average uncertainty of $\sim 30 \%$ (see Fig. 23 of Elbaz et al. 2011). Therefore, this does not introduce any bias in our results. To determine the dust temperature, we fitted the observational data with a modified black body (MBB) model by fixing the emissivity parameter to $\beta=1.5$. We required at least one flux measurement at each "Wien" and "Rayleigh-Jeans" side of the FIR peak (i.e., at least two measurements in total; see Hwang et al. 2010b for detailed selection criteria). This left us 284 and 104 galaxies with $T_{\text {dust }}$ measurements in GOODS-N and -S, respectively.

\subsection{Sloan digital sky survey sample}

For local galaxies, we used a spectroscopic sample of galaxies in the SDSS Data Release 7 (Abazajian et al. 2009, SDSS DR7) complemented by a photometric sample of SDSS galaxies, whose redshift information is not available in the SDSS database, but available in the literature (Hwang et al. 2010a). In addition, we used the FIR data for these galaxies compiled in Hwang et al. (2010b) by cross-correlating IRAS Faint Sources Catalog - Version 2 (Moshir et al. 1992) and AKARI/Far-Infrared Surveyor (FIS; Kawada et al. 2007) allsky survey Bright Source Catalogue $\left(\mathrm{BSC}^{1}\right)$ ver. 1.0 with the SDSS samples.

\footnotetext{
${ }^{1}$ http://www.ir.isas.jaxa.jp/AKARI/Observation/PSC/ Public/RN/AKARI-FIS_BSC_V1_RN.pdf
} 
The $r$-band absolute magnitude $M_{r}$ was also computed in fixed bandpasses, shifted to $z=0.1$, using Galactic reddening correction (Schlegel et al. 1998) and $K$-corrections (Blanton \& Roweis 2007). The evolution correction given by Tegmark et al. (2004), $E(z)=1.6(z-0.1)$, was also applied. Note that the amount of evolution correction is different between GOODS and SDSS galaxies. These values are taken from the redshift evolution of the characteristic luminosity in the luminosity function of local and high-z galaxies separately, so we kept different values. Change of these values does not affect our conclusions.

By adopting a method similar to the one applied to GOODS galaxies, we computed the IR luminosity of 14444 galaxies (among the total sample of 926,748 SDSS galaxies) whose IRAS $60 \mu \mathrm{m}$ or AKARI $90 \mu \mathrm{m}$ flux densities are reliable $^{2}$ using the CE01 SED templates by allowing normalization of the templates. We fitted the flux densities without allowing the normalization of the templates if there were two or less data points to fit. To determine the dust temperatures, we again fitted the observational data with a modified black body (MBB) model by fixing the emissivity parameter to $\beta=1.5$ only for 238 galaxies detected at AKARI 140 or $160 \mu \mathrm{m}$ so that we were able to obtain flux density measurements longward of the FIR peak as well as the one shortward of the peak in a similar way to GOODSHerschel galaxies (see Hwang et al. 2010b for detailed selection criteria).

The stellar mass estimates were obtained from the MPA/JHU DR7 value-added galaxy catalog 3 (VAGC), which are based on the fit of SDSS five-band photometry (Kauffmann et al. 2003; Gallazzi et al. 2005). We converted these estimates based on Kroupa IMF (Kroupa 2001) to a Salpeter IMF by dividing them by a factor of 0.7 (Elbaz et al. 2007).

We adopted the galaxy morphology information from the Korea Institute for Advanced Study (KIAS) DR7 VAGC ${ }^{4}$ (Park \& Choi 2005; Choi et al. 2010). We performed additional visual classification for the galaxies in the SDSS database that are not included in KIAS DR7 VAGC.

\subsection{Comparison of GOODS and SDSS galaxies}

In the right panels of Fig. 1 we plot several physical parameters of the GOODS galaxies as a function of redshift, and define a volume limited sample to be analyzed $\left(-19.5 \geq M_{r}\right.$ with $0.4 \leq z \leq 1.2)$. Similarly, we plot the SDSS galaxies in the left panels of Fig. 1, and define a volume limited sample of galaxies with $-19.5 \geq M_{r}$ and $0.005 \leq z \leq 0.0656$. The comoving volume for this SDSS sample is $\sim 2.0 \times 10^{7} \mathrm{Mpc}^{3}$, which is much larger than that for the GOODS galaxies $\left(\sim 2.4 \times 10^{5} \mathrm{Mpc}^{3}\right.$ for GOODS-N at $0.4 \leq z \leq 1.2$ ). Note that the FIR detection limits for local SDSS and high- $z$ GOODS galaxies are not the same even if we fix the mass and luminosity of galaxies in Sect. 3. However, because we are interested in the difference of SFA depending on the morphology of and the distance to the nearest neighbor galaxy in a given redshift range, the different FIR detection limits between local and high- $z$ galaxies do not affect our conclusions.

In Fig. 2 we plot the dust temperature versus FIR flux density ratio $\left(S_{60, \text { IRAS }} / S_{100, \text { IRAS }}\right)$ for SDSS galaxies, which shows a good correlation between the two quantities (see also Chanial et al. 2007). From the fit with an ordinary least-squares bisector

\footnotetext{
2 Flux quality flags are either "high" or "moderate" for IRAS sources and "high" for AKARI sources.

${ }^{3}$ http://www.mpa-garching.mpg.de/SDSS/DR7/

${ }^{4}$ http://astro.kias.re.kr/vagc/dr7/
}

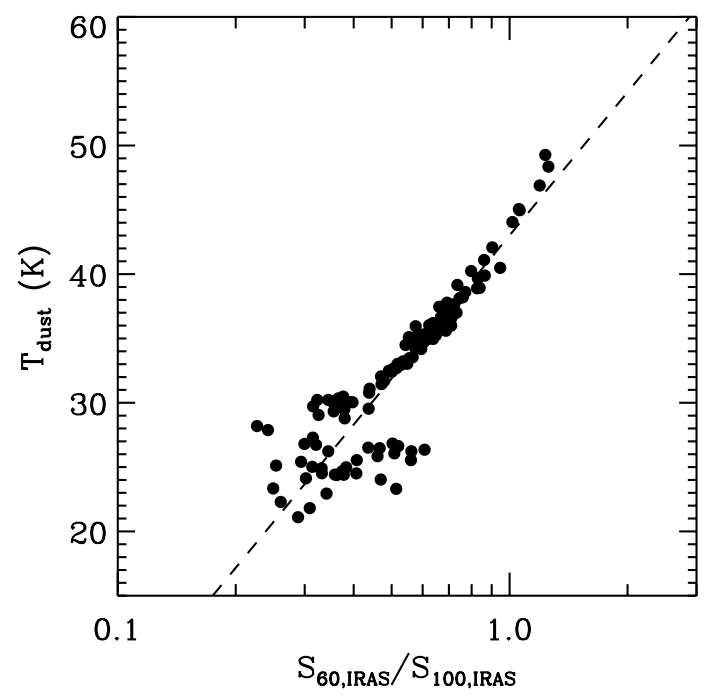

Fig. 2. Correlation between dust temperature $\left(T_{\text {dust }}\right)$ and flux density ratios $\left(S_{60, \text { IRAS }} / S_{100, \text { IRAS }}\right)$ for local SDSS galaxies. The dashed line represents the best fit with an ordinary least-squares bisector method.

method (Isobe et al. 1990), we derive a transformation relation between the two quantities,

$T_{\text {dust }}(\mathrm{K})=(43.0 \pm 0.3)+(37.0 \pm 1.5) \log \left(S_{60, \text { IRAS }} / S_{100, \text { IRAS }}\right)$.

Because the number of SDSS galaxies with $S_{60 \text {,IRAS }} / S_{100 \text {,IRAS }}$ is larger than that with $T_{\text {dust }}$ measurements, we transformed the FIR flux density ratio into the dust temperature using this equation to increase the statistics (see Fig. 6).

\subsection{Nearest neighbor galaxy}

To investigate the effects of interactions with the nearest neighbor galaxy, we first identified the nearest neighbor of a target galaxy that is the closest to the target galaxy on the projected sky and that satisfies the conditions of magnitude and relative velocity. We searched for the nearest neighbor galaxy among galaxies that have magnitudes brighter than $M_{r}=M_{r \text {,target }}+0.5$ and have relative velocities less than $\Delta v=\left|v_{\text {neighbors }}-v_{\text {target }}\right|=660 \mathrm{~km} \mathrm{~s}^{-1}$ for early-type target galaxies and less than $\Delta v=440 \mathrm{~km} \mathrm{~s}^{-1}$ for late-type target galaxies. Because the redshift uncertainties are larger for the GOODS galaxies, we use velocity difference limits that are $10 \%$ larger than those that we have allowed for SDSS galaxies in previous studies $\left(\Delta v=600\right.$ and $400 \mathrm{~km} \mathrm{~s}^{-1}$ for early- and late-type target SDSS galaxies, respectively, as seen in Fig. 1 of Park et al. 2008). The use of different values for the relative velocity condition depending on galaxy morphology is supported by different velocity distributions of neighboring galaxies depending on galaxy morphology, as shown in Fig. 1 of Park et al. (2008) and Fig. 2 of Park \& Choi (2009) for SDSS galaxies and Fig. 3 of Hwang \& Park (2009) for GOODS galaxies. Because we used volume-limited samples of galaxies with $M_{r} \leq-19.5$, we restrict our analysis to target galaxies brighter than $M_{r \text {,target }}=-20$ so that their neighbors $\left(M_{r \text {,nei }} \leq M_{r \text {,target }}+0.5\right)$ are searched within the volume-limited samples.

The virial radius of a galaxy within which the mean mass density is 200 times the critical density of the universe $\left(\rho_{\mathrm{c}}\right)$, is calculated by

$r_{\mathrm{vir}}=(3 \gamma L / 4 \pi)^{1 / 3}\left(200 \rho_{\mathrm{c}}\right)^{-1 / 3}$,

where $L$ is the galaxy luminosity, and $\gamma$ the mass-to-light ratio. Here, the mass associated with a galaxy plus dark halo 
system is assumed to be proportional to the $r$-band luminosity of the galaxy. We assume that the mass-to-light ratio of early-type galaxies is on average twice as high as that of latetype galaxies at the same absolute magnitude $M_{r}$, which means $\gamma($ early $)=2 \gamma($ late) (see Sect. 2.5 of Park \& Choi (2009) and Sect. 2.4 of Park et al. (2008) for SDSS galaxies, and Sect. 2.3 of Hwang \& Park (2009) for GOODS galaxies). The critical density of the universe $\rho_{\mathrm{c}}$ is a function of redshift $z$ [i.e. $\left.\rho_{\mathrm{c}}=3 H^{2}(z) /(8 \pi G)\right]$ and $\Omega_{\mathrm{m}}(z)=\rho_{b}(z) / \rho_{\mathrm{c}}(z)=\bar{\rho}(1+z)^{3} / \rho_{\mathrm{c}}(z)$, where $\rho_{b}$ and $\bar{\rho}$ are the mean matter densities in proper and comoving spaces, respectively ${ }^{5}$. The Hubble parameter at $z$ is $H^{2}(z)=H_{0}^{2}\left[\Omega_{\mathrm{m}, 0}(1+z)^{3}+\Omega_{k, 0}(1+z)^{2}+\Omega_{\Lambda, 0}\right]$, where $\Omega_{\mathrm{m}, 0}$, $\Omega_{k, 0}$, and $\Omega_{\Lambda, 0}$ are the dimensionless density parameters at the present epoch (Peebles 1993). Then, the virial radius of a galaxy at redshift $z$ in proper space can be rewritten by

$r_{\mathrm{vir}}(z)=\left[3 \gamma L \Omega_{\mathrm{m}, 0} /(800 \pi \bar{\rho}) /\left\{\Omega_{\mathrm{m}, 0}(1+z)^{3}+\Omega_{k, 0}(1+z)^{2}+\Omega_{\Lambda, 0}\right\}\right]^{1 / 3}$.

The value of mean density of the universe for local SDSS galaxies, $\bar{\rho}=(0.0223 \pm 0.0005)(\gamma L)_{-20}\left(h^{-1} \mathrm{Mpc}\right)^{-3}$, was adopted, where $(\gamma L)_{-20}$ is the mass of a late-type galaxy with $M_{r}=-20$ (Park et al. 2008).

For high- $z$ GOODS galaxies, we computed the mean mass density $\bar{\rho}$ using the galaxies at $z=0.4-1.2$ with various absolute magnitude limits varying from $M_{r}=-16$ to -20 . We found that the mean mass density appears to converge when the magnitude cut is fainter than $M_{r}=-17.5$, which means that the contribution of faint galaxies is not significant because of their small masses. In this calculation, each galaxy is weighted by the inverse of completeness according to its apparent magnitude and color (see Fig. 1 of Hwang \& Park 2009). We obtain $\bar{\rho}=0.017$ and $0.013(\gamma L)_{-20}\left(\mathrm{Mpc}^{-3}\right)$ for GOODS-N and $-\mathrm{S}$, respectively, where $(\gamma L)_{-20}$ is the mass of a late-type galaxy with $M_{r}=-20$. According to our formula the virial radii of galaxies with $M_{r}=-20$ and -21 are 300 and $400 h^{-1}$ kpc for early types, and 240 and $320 h^{-1} \mathrm{kpc}$ for late types, respectively.

The spectroscopic completeness can affect the identification of the genuine nearest neighbor, and then the nearest neighbor can be seriously misidentified if the completeness is very low. Our previous Monte Carlo experiment shows that the fraction of the misidentified nearest neighbor reaches about 50\% when the sample completeness is 50\% (Hwang \& Park 2009). Therefore, it is necessary to have survey data with high completeness so that one does not miss the genuine nearest neighbor. Up to now, GOODS has the highest spectroscopic completeness (71-86\% for GOODS galaxies at $m_{z}<23$ ) among several extensive, deepfield surveys with HST images to our knowledge. Therefore, GOODS is the best survey data for our analysis, but it should be noted that our results could be weakened by this incompleteness. The completeness depends on the apparent magnitude and color (see Fig. 1 of Hwang \& Park 2009) and also on the distance between galaxies owing to the difficulty in observing galaxies close to each other with the multiobject spectrograph (MOS). We checked the completeness as a function of the projected distance to the target galaxy, and found that it does not change with the projected distance. It might be because we combined spectroscopic data from numerous references, therefore, the difficulty in observing galaxies with small separation using MOS is significantly reduced. Similarly, the redshift information of some SDSS galaxies missed by the SDSS database was complemented

\footnotetext{
5 We correct a typo for $\Omega_{\mathrm{m}}(z)$ in Hwang \& Park (2009), but all the related values in that paper are correct.
}

by the data in the literature (Hwang et al. 2010a), so there is no bias for local galaxies, either.

Note that the nominal FWHMs of the point spread function (PSF) are 37" $(65 \mu \mathrm{m}), 39^{\prime \prime}(90 \mu \mathrm{m}), 58^{\prime \prime}(140 \mu \mathrm{m})$, and $61^{\prime \prime}(160 \mu \mathrm{m})$ for AKARI bands (Kawada et al. 2007), and $1.44^{\prime}(60 \mu \mathrm{m})$ and $2.94^{\prime}(100 \mu \mathrm{m})$ for IRAS bands (Sanders et al. 2003). The corresponding angular size of one viral radius for typical late- and early-type galaxies with $M_{r}=-21$ and $z=$ 0.035 , is $11.3^{\prime}$ and $14.3^{\prime}$, respectively. Therefore, though the galaxies in pairs are selected from galaxy catalogs in optical bands with high spatial resolutions, the measured SFRs of galaxies in close pairs cannot be clearly assigned to the individual galaxies because of the poor spatial resolution of FIR data. For example, local galaxies with $M_{r}=-21$ and $z=0.035$ having neighbors at $\lesssim 0.06 r_{\text {vir }}\left(\$ 0.12 r_{\text {vir }}\right)$ are not resolved with AKARI $90 \mu \mathrm{m}$ (IRAS $60 \mu \mathrm{m}$ ), because the pair separation is smaller than the FWHM at each band. Consequently, the measured SFRs for these galaxies can indeed indicate SFRs of the whole interacting systems. However, our results on the increased SFRs (or SSFRs) caused by galaxy-galaxy interactions that we present in Sect. 3 are not strongly affected by this effect because the increased SFRs (or SSFRs) are found to be much higher than a factor of two, which could be simply caused by this blending problem of two galaxies in one FIR beam.

For high-z galaxies, the PSF FWHMs are 6.0" (Spitzer $24 \mu \mathrm{m})$, 6.7" (Herschel $100 \mu \mathrm{m}), 11.0^{\prime \prime}(160 \mu \mathrm{m}), 18.1^{\prime \prime}$ (250 $\mu \mathrm{m}), 24.9^{\prime \prime}(350 \mu \mathrm{m})$, and 36.6" $(500 \mu \mathrm{m})$. The angular size of one virial radius for typical late- and early-type galaxies with $M_{r}=-21$ would be, respectively, $70.8^{\prime \prime}$ and $88.6^{\prime \prime}$ at $z=0.6$, and 59.1" and 73.5" at $z=1.0$. Similar to the case of local galaxies, high- $z$ galaxies having neighbors at $\$ 0.07 r_{\text {vir }}$ $\left(\$ 0.09 r_{\text {vir }}\right)$ are not resolved with Spitzer $24 \mu \mathrm{m}$ (source extraction on Herschel images was performed at the prior positions of Spitzer $24 \mu \mathrm{m}$-selected sources; Elbaz et al. 2011). However, this has no effect on our conclusions.

\section{Results}

\subsection{Change in SSFRs as a function of pair separation}

In Fig. 3 we plot several FIR properties of local (left) and high$z$ (right) late-type galaxies as a function of the projected distance to the nearest neighbor galaxy normalized by the virial radius of the neighbor $\left(R_{\mathrm{n}} / r_{\mathrm{vir}, \text { nei }}\right)$. Note that we only plot IRAS or AKARI-selected (left) and Herschel-selected (right) late-type galaxies, but their nearest neighbors are selected among the spectroscopic samples of SDSS and GOODS galaxies regardless of FIR detections. To remain complete in terms of neighbor galaxies and remove the effect of mass on the SFA, we restrict our analysis to the late-type galaxies with $-20 \geq M_{r}$ and $10^{10} \leq M_{\text {star }}\left(M_{\odot}\right) \leq 10^{11}$. Therefore, even if we use the physical distance for the pair separation without using a normalization by the virial radius, the observed trends in this figure do not change.

For high- $z$ samples, we only plot GOODS galaxies that have at most one neighbor within $6^{\prime \prime}$ (the full width half maximum, $F W H M$, of the Spitzer beam at $24 \mu \mathrm{m}$ ) with $S_{24}>50 \%$ of the central $24 \mu \mathrm{m}$ source (i.e. $N_{\text {nei, } 24 \mu \mathrm{m}} \leq 1$ ). This criterion is introduced to reduce the contamination of neighboring sources, but to allow for the possibility of having one close neighbor to study the effects of galaxy proximity. In practice, this criterion results in the removal of only four galaxies among 330 GOODS galaxies in Fig. 3, which has no effect on our conclusions.

Assuming a Salpeter IMF, we converted the IR luminosity into SFR $_{I R}$ using the relation in Kennicutt (1998): SFR $_{I R}$ 

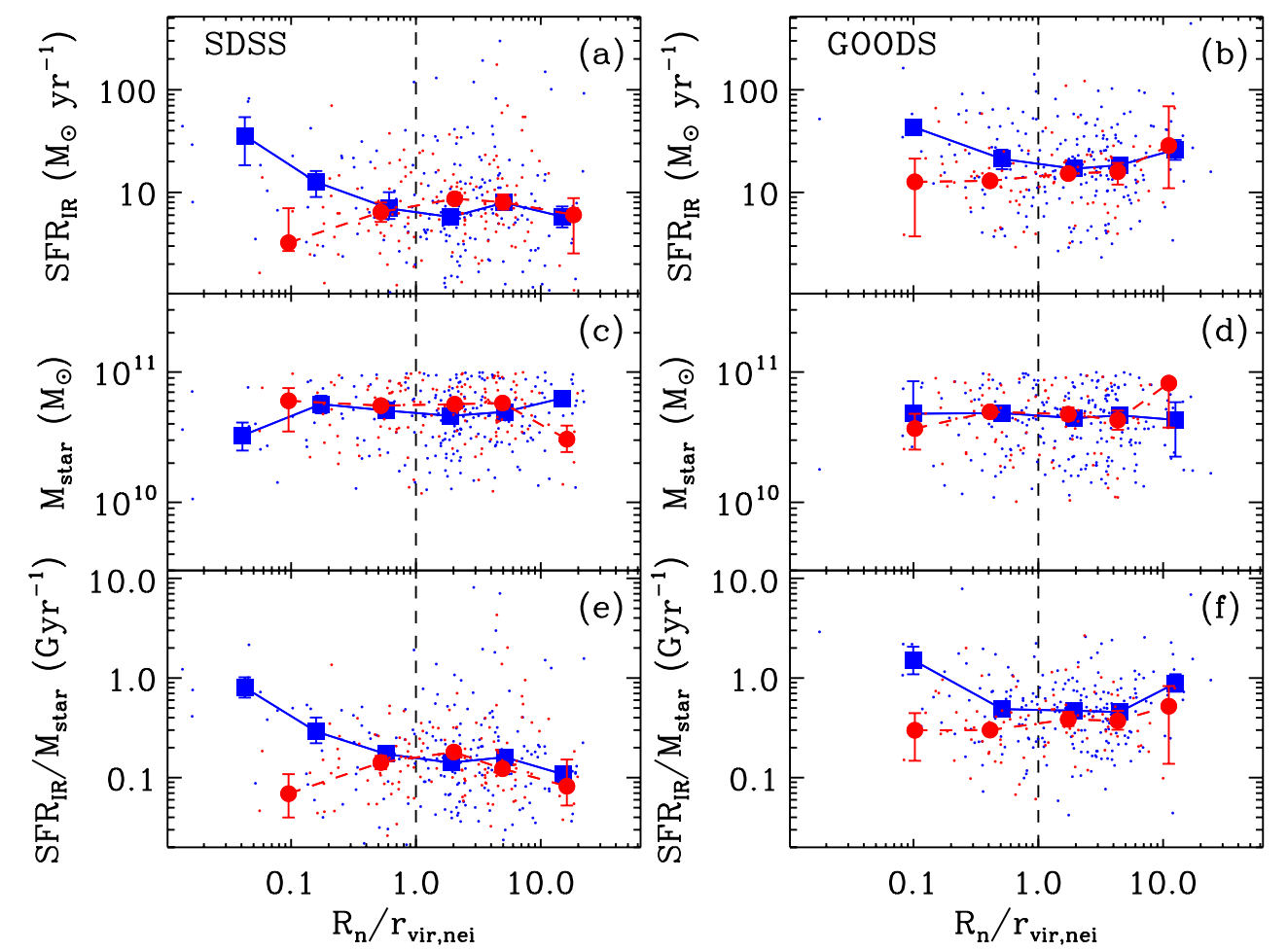

Fig. 3. (a)-b)) SFR, (c)-d)) $M_{\text {star }}$, (e)-f)) specific SFR for late-type galaxies with $-20 \geq M_{r}$ and $10^{10} \leq M_{\text {star }}\left(M_{\odot}\right) \leq 10^{11}$ as a function of the projected distance to the nearest neighbor galaxy $\left(R_{\mathrm{n}} / r_{\text {vir,nei }}\right)$ in (left) SDSS $(0.005 \leq z \leq 0.0656)$ and (right) GOODS $\left(0.4 \leq z_{\text {spec }} \leq 1.2\right)$. Red and blue dots are late-type galaxies with early- and late-type nearest neighbor galaxies, respectively. Large circle (early-type neighbor case) and square (late-type neighbor case) are median values of each physical parameter and of $R_{\mathrm{n}} / r_{\mathrm{vir}, \text { nei }}$ at each distance bin. The errorbars represent $68 \%$ $(1 \sigma)$ confidence intervals that are determined by the bootstrap resampling method.

$\left(M_{\odot} \mathrm{yr}^{-1}\right)=1.72 \times 10^{-10} L_{\mathrm{IR}}\left(L_{\odot}\right)$. Panels $(\mathrm{a}-\mathrm{b})$ show that $\mathrm{SFR}_{\mathrm{IR}}$ depends on the projected distance to the nearest neighbor as well as on the neighbor's morphology. Large circle (early-type neighbor case) and square (late-type neighbor case) are median values of each physical parameter and of $R_{\mathrm{n}} / r_{\mathrm{vir}, \mathrm{nei}}$ at each distance bin. Obviously, the $\mathrm{SFR}_{\mathrm{IR}}$ changes with neither the distance to the neighbor nor the neighbor's morphology when a galaxy is located farther than $0.5 r_{\text {virnei }}$ (virial radius of neighbor galaxy). On the other hand, when a galaxy is located at $R_{\mathrm{n}} \lesssim 0.5 r_{\text {vir,nei }}$, the $\mathrm{SFR}_{\mathrm{IR}}$ increases as the target, late-type galaxy approaches a late-type neighbor, but decreases or remains constant as it approaches an early-type neighbor. It is important to note that the bifurcation of SFR $\mathrm{IR}$ depending on the neighbor's morphology occurs at $R_{\mathrm{n}} \approx 0.5 r_{\text {vir,nei }}$.

Because the SFR is strongly correlated with the mass (Brinchmann et al. 2004; Elbaz et al. 2007; Daddi et al. 2007; Magdis et al. 2010c), it is important to check the effects of galaxy mass on the change of SFR. Therefore we plot the distributions of stellar masses and specific SFRs ( $\mathrm{SFR}_{\mathrm{IR}} / \mathrm{M}_{\text {star }}$, SSFRs) in panels (c-f). Panels (c, d) show no significant change of masses as a function of $R_{\mathrm{n}}$, but panels $(\mathrm{e}, \mathrm{f})$ suggest that a difference in SSFRs between early- and late-type neighbor cases is still prominent at $R_{\mathrm{n}} \lesssim 0.5 r_{\text {vir,nei }}$. We also checked the redshift distributions as a function of $R_{\mathrm{n}}$ (not shown), which again shows no significant dependence on $R_{\mathrm{n}}$ and the morphology of the neighbor. We used a Kolmogorov-Smirnov (K-S) test to determine whether the SSFR distributions of interacting and noninteracting galaxies with late-type neighbors are drawn from the same distribution. We tested two cases of interacting galaxies with late-type neighbors: 1$)$ strongly interacting $\left(R_{\mathrm{n}} \leq 0.1 r_{\text {vir,nei }}\right)$ galaxies versusnon-interacting galaxies $\left(R_{\mathrm{n}}>r_{\mathrm{vir}, \mathrm{nei}}\right)$; and 2$)$ relatively weakly interacting $\left(R_{\mathrm{n}} \leq 0.5 r_{\text {vir,nei }}\right)$ galaxies versus non-interacting galaxies. The hypothesis that the two distributions are extracted from the same parent population can be rejected at a confidence level of $>99 \%$ for both cases in the local universe. In the high- $z$ universe, the hypothesis can be rejected at a confidence level of $>99$ and $90 \%$ for strongly and weakly interacting galaxies, respectively. To help the understanding of the difference in SSFRs for several subsamples, we plot in Fig. 4 the distribution of SSFRs for interacting and non-interacting galaxies in SDSS and GOODS.

The statistical significance of the increased SSFRs of interacting galaxies with late-type neighbors compared to noninteracting galaxies was also tested with a Monte Carlo (MC) test. We constructed two subsamples (with the same number of galaxies as in the actual samples) by randomly drawing the SSFRs from the whole galaxy sample and computed the median of each subsample. The resulting two subsamples will have the same medians on average. These random subsamples will tell us whether or not the difference in SSFRs between interacting and non-interacting galaxies with late-type neighbors is statistically significant. We constructed 1000 trial data sets and computed the fraction of simulated data sets in which the difference of the SSFRs is larger than or equal to that based on the real data $\left(f_{\text {sim } \geq o b s}\right)$. The significance levels of the difference defined by $100\left(1-f_{\text {sim } \geq \text { obs }}\right)(\%)$. This test also confirms the increased SSFRs of interacting galaxies with late-type neighbors compared to non-interacting galaxies with a significance level of $>99 \%$ for both strongly and weakly interacting galaxies in local and high- $z$ universe.

Similarly, the statistical significance of different SSFR distributions of interacting galaxies with early- and late-type neighbors at $R_{\mathrm{n}} \leq 0.5 r_{\text {vir,nei }}$ is examined with the K-S and MC tests. The difference is confirmed with a significance level of 98 and 
H. S. Hwang et al.: GOODS-Herschel: the impact of galaxy-galaxy interactions on the far-infrared properties of galaxies
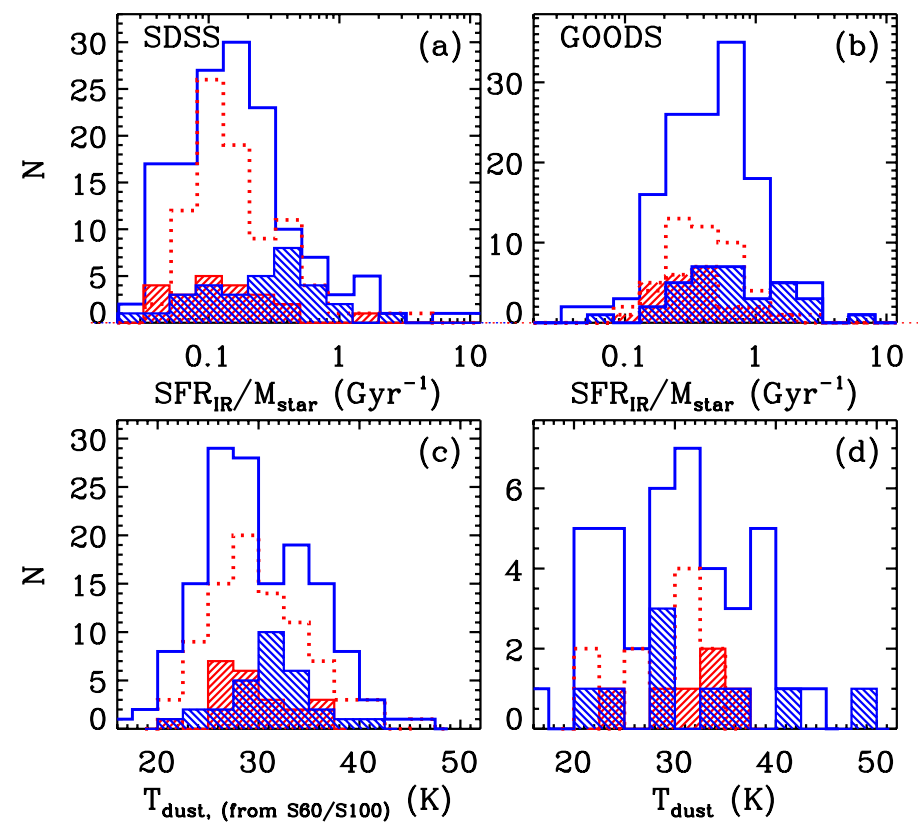

Fig. 4. Distribution of SSFRs (top) and $T_{\text {dust }}$ (bottom) for SDSS (left) and GOODS (right) galaxies. Non-interacting galaxies (i.e. $R_{\mathrm{n}}>r_{\text {virnei }}$ in Fig. 3) with early- and late-type neighbors are shown by solid and dotted histograms, respectively. Interacting galaxies (i.e. $R_{\mathrm{n}} \leq 0.5 r_{\text {vir,nei }}$ ) with early- and late-type neighbors are denoted by hatched histograms with orientation of $45^{\circ}$ (// with red color) and of $315^{\circ}$ ( $\backslash \backslash$ with blue color) relative to horizontal, respectively.

$>99 \%$ from the K-S and MC tests, respectively, in the local universe, and of $98 \%$ from both K-S and MC tests in high- $z$ universe.

To study the evolution of SSFRs depending on the morphology of the nearest neighbor, we plot in Fig. 5 the median SSFRs of strongly and weakly interacting galaxies as a function of redshift in comparison with those for non-interacting galaxies. We plot the SSFRs of interacting galaxies normalized by those of non-interacting galaxies at each redshift bin to focus only on the relative ratio of SSFRs of interacting galaxies to those of noninteracting galaxies.

For the early-type neighbor case (left panel in Fig. 5), the SSFRs of interacting galaxies are similar to or slightly smaller than those of non-interacting galaxies in all redshift bins. However, for the late-type neighbor case (right panel), obviously the increased SSFRs for weakly and strongly interacting galaxies are systematically higher than those for non-interacting galaxies in all redshift bins. The SSFRs of strongly and weakly interacting galaxies are, on average, higher than those of noninteracting galaxies by factors of about $4.0 \pm 1.2$ and $1.8 \pm 0.7$, respectively. If these increased SSFRs are simply caused by the blending of two galaxies with similar masses in one FIR beam without any enhanced SFA, the SSFRs are expected to increase by a factor of only two. Indeed, the neighbor-separation scale of $R_{\mathrm{n}} \sim 0.05 r_{\text {vir,nei }}\left(\approx 15 h^{-1} \mathrm{kpc}\right.$ for galaxies at $\left.M_{r}=-21\right)$ is important because the galaxies in pairs start to merge at this separation, so the SFA starts to change abruptly (Park \& Choi 2009). Thus the increased SFR for strongly interacting galaxies $\left(R_{\mathrm{n}} \leq 0.1 r_{\text {vir,nei }} \approx 30 h^{-1} \mathrm{kpc}\right.$ for $\left.M_{r}=-21\right)$ by a factor of four really reflects the enhancement of SFA caused by a merger.

\subsection{Change in dust temperature as a function of pair separation}

In Fig. 6 we show the dependence of dust temperature on the distance to and the morphology of the nearest neighbor galaxy. For local SDSS galaxies in (a), we plot the dust temperature derived from the flux density ratio $\left(S_{60} / S_{100}\right)$ using Eq. (1) to increase the statistics. For high-z GOODS galaxies in (b), we do not use the dust temperature derived from the FIR flux density ratio because the large scatter in the correlation between Herschel flux density ratios and dust temperature makes statistics worse.

The dust temperature of both local and high-z late-type galaxies appears to increase as they approach late-type neighbors, which is clearly seen at $R_{\mathrm{n}} \lesssim 0.1 r_{\text {vir,nei }}$. We also show the distribution of dust temperature in Fig. 4 for interacting and noninteracting galaxies in SDSS and GOODS. The MC test supports this different $T_{\text {dust }}$ distribution between strongly interacting and non-interacting galaxies with late-type neighbors with a significance level of 96 and $>99 \%$ for local and high- $z$ galaxies, respectively. However, there are only two GOODS galaxies at $R_{\mathrm{n}} \lesssim 0.1 r_{\text {vir,nei }}$, so this trend needs to be checked with more extended data sets in future studies. On the other hand, the dust temperature of local late-type galaxies with early-type neighbors at $R_{\mathrm{n}} \lesssim 0.1 r_{\text {vir,nei }}$ seems to be lower than or marginally similar to that of non-interacting galaxies. Therefore, the difference in the dust temperature of strongly interacting galaxies with lateand early-type neighbors seems to be significant, which is supported by the MC test with a significance level of $98 \%$. For high$z$ galaxies, there are few late-type galaxies with close early-type neighbors at $R_{\mathrm{n}} \lesssim 0.1 r_{\text {vir,nei }}$ with $T_{\text {dust }}$ measurements, so we cannot study the trend.

\section{Discussion}

The increase of SSFR for galaxies in pairs found in this study is consistent with results in previous studies at low redshifts (e.g., Kennicutt et al. 1987; Barton et al. 2000; Lambas et al. 2003; Nikolic et al. 2004; Woods \& Geller 2007; Huang \& Hwang 2011) and high redshifts (e.g., Lin et al. 2007; Wong et al. 2011). However, note that the amount of increased SSFR compared to isolated galaxies is different depending on the studies because the definition of interacting galaxies and the observational selection effects are different. The increase of dust temperature in local late-type galaxies that strongly interact with other late-type neighbors is also consistent with previous studies in the sense that the contribution of warm dust compared to cold dust increases with the merging sequence (Telesco et al. 1988; Xilouris et al. 2004). However, we found the hint of this trend for high- $z$ galaxies at $0.4 \lessgtr z \lessgtr 1.2$ for the first time. It is also interesting to see that the dust temperature of local, late-type galaxies does not seem to increase when they are strongly interacting with earlytype neighbors (see Fig. 6). Note also that the distance to the nearest neighbor might not be a direct measure of the merging sequence because galaxies in pairs would merge after several encounters and their orbital geometry is complicated.

We wish to emphasize that our analysis represents a major improvement compared to previous studies because we 1) have robust SFR measurements of galaxies with well-constrained FIR SEDs thanks to Herschel and AKARI; 2) have galaxy samples with high spectroscopic completeness (i.e. $>85 \%$ for SDSS galaxies at $m_{r}<17.77$ and $71-86 \%$ for GOODS galaxies at $m_{z}<23$ ); 3) study only the galaxies that are complete in terms of neighbor galaxies with stellar masses similar to target galaxies $\left(M_{r \text {,nei }} \leq M_{r \text {,target }}+0.5\right)$; 4) distinguish the morphology 


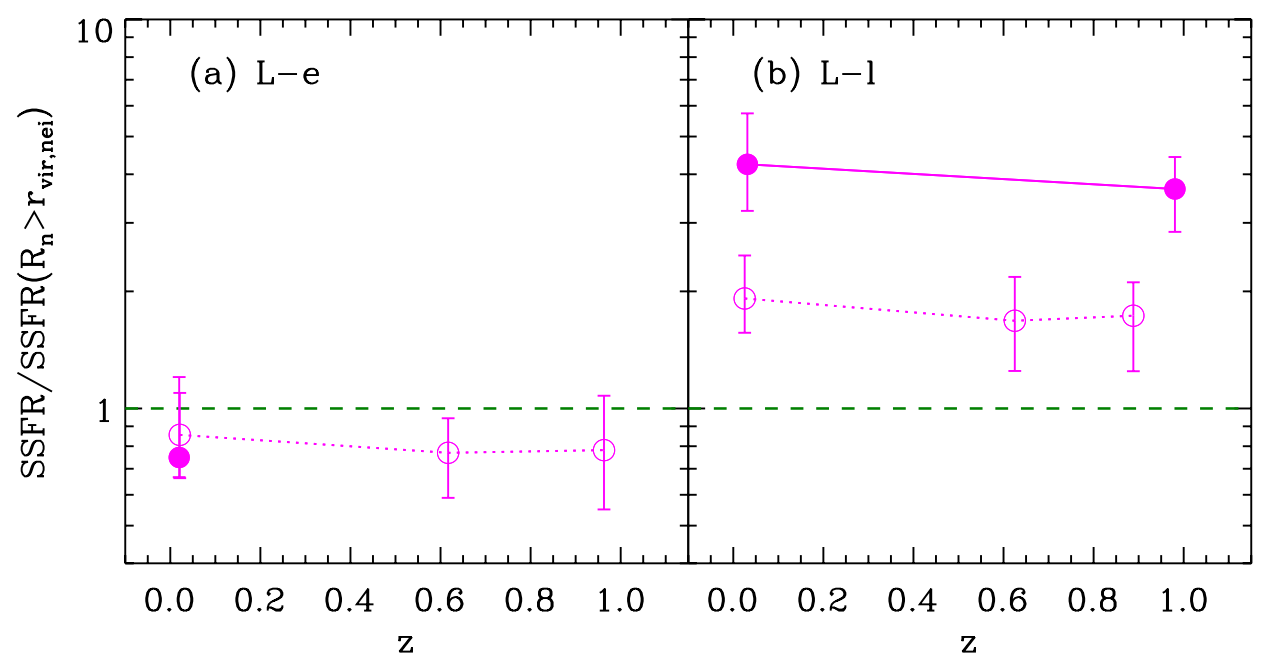

Fig. 5. SSFRs of interacting galaxies normalized by SSFRs of non-interacting galaxies (i.e. $R_{\mathrm{n}}>r_{\text {virnei }}$ in Fig. 3) as a function of redshift for a) early-type and b) late-type neighbor case. Filled and open circles are median values for strongly (i.e. $R_{\mathrm{n}} \leq 0.1 r_{\text {virnei }}$ ), and weakly interacting (i.e. $\left.R_{\mathrm{n}} \leq 0.5 r_{\text {vir,nei }}\right)$ galaxies, respectively, at each redshift bin $(0.005 \leq z \leq 0.0656,0.4 \leq z \leq 0.8$, and $0.8 \leq z \leq 1.2)$.
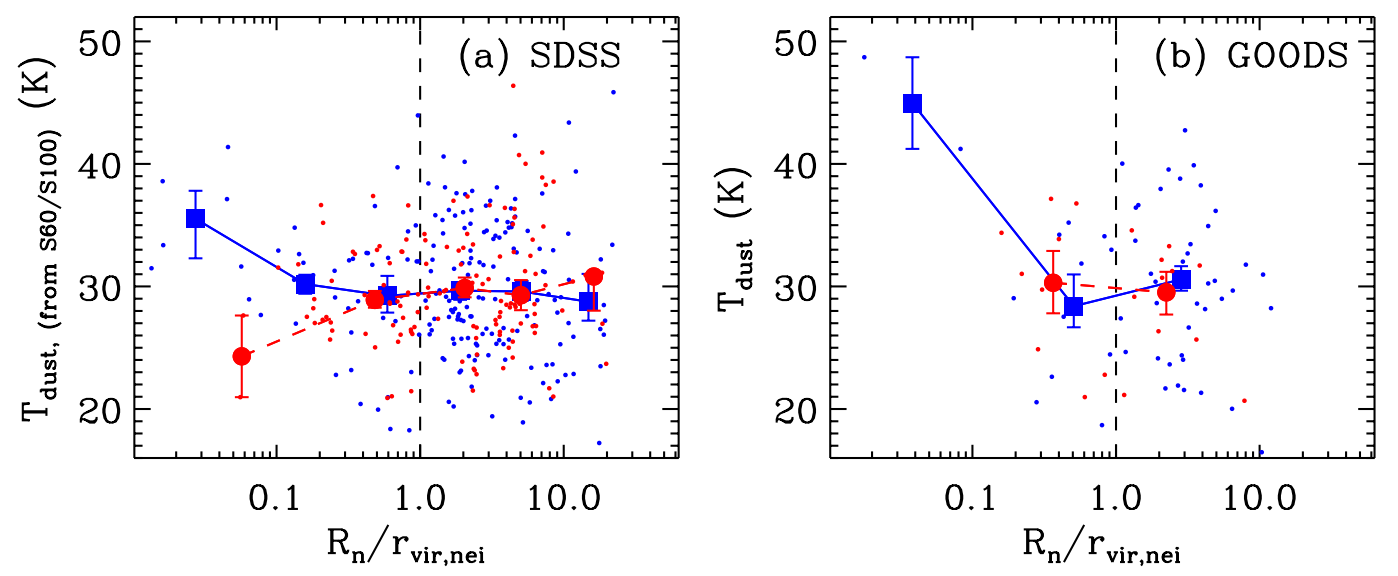

Fig. 6. Same as Fig. 3, but for the dust temperature of a) SDSS and b) GOODS galaxies.

of galaxies in pairs, which is an important indicator of the gas fraction in galaxies; and 5) use a distance to the nearest neighbor normalized by the virial radius of the neighbor instead of the physical distance to account for different masses and corresponding crossing time of galaxy samples.

The dependence of SFR (or SSFR) on the morphology of the neighbor at $R_{\mathrm{n}} \approx 0.5 r_{\text {vir,nei }}$ seen in Fig. 3 may imply that the hydrodynamic interactions with the nearest neighbor play critical roles in triggering the SFA of galaxies in addition to the tidal interactions. This dependence is also observed for local normal galaxies (Park \& Choi 2009; Xu et al. 2010) and local LIRGs and ULIRGs (Hwang et al. 2010a), and can be explained as follows. If a late-type galaxy approaches a late-type neighbor within half of the virial radius of the neighbor, the cold gas inflow into the central region of the target galaxy from the neighbor galaxy as well as from the disk of the target galaxy increases, which results in the enhanced SFA of the target galaxy. Because of this starburst mode of compact star formation, the SSFR and the dust temperature are expected to increase as we observed (Elbaz et al. 2011; see also Chanial et al. 2007). Then when two galaxies finish merging, the end product of the merger will be bright because of the very recent SFA, and the new nearest neighbor galaxy of the merger product will be far away. This may explain the existence of some non-interacting galaxies at $R_{\mathrm{n}}>r_{\mathrm{vir}, \mathrm{nei}}$ with high SSFRs and $T_{\text {dust }}$ as seen in Figs. 3 and 6.

On the other hand, if a galaxy approaches an early-type neighbor within the virial radius of the neighbor, the hot gas of the early-type neighbor prevents the galaxy from forming stars with cold gas, and/or there is no inflow of cold gas from its earlytype neighbor, so the SFA of the galaxy is not boosted even if it does have a close companion. This may explain why not all mergers are in a starburst mode. The SF quenching mechanisms of hot gas in early-type neighbors could be similar to those of a hot intracluster medium of galaxy clusters acting on late-type galaxies in it, which are hydrodynamic processes such as thermal evaporation, strangulation, ram pressure stripping, or viscous stripping (Park \& Choi 2009; Park \& Hwang 2009). Indeed, the X-ray observations of galaxies in pairs with mixed morphology show evidence for extended X-ray halos of the early type that surround the late type, which supports our interpretation (Grützbauch et al. 2007). In addition, that the SSFRs in Fig. 5 are increased by similar factors in all redshift bins suggests that similar physical mechanisms (such as hydrodynamic interactions plus tidal interactions described above) have affected the SFA over at least 8 billion years. 
The SFRs of galaxies are known to depend strongly on the local density in the sense that spatially averaged SFRs of galaxies or star-forming galaxy fractions decrease as the background density increases in the local universe (e.g., Lewis et al. 2002; Gómez et al. 2003; Park et al. 2007; Lee et al. 2010; Hwang et al. 2010a). In the high- $z$ universe, some studies found similar results (e.g., Patel et al. 2009; Feruglio et al. 2010), but there are also hints for an opposite trend (i.e. increasing SFRs or star-forming galaxy fractions with increasing the background density) known as the reversal of SFR-density relation (e.g., Elbaz et al. 2007; Popesso et al. 2011a,b). When we consider the morphology-density relation (Dressler 1980), early(late)-type neighbors could be preferentially selected in high(low)-density regions, and the pair separation is correlated with the local density. Therefore, the SFRs appear to depend on both large- and small-scale (attributed to the nearest neighbor) environments. One might expect that the difference in FIR properties depending on the morphology of and the distance to the nearest neighbor is simply caused by the statistical correlation between the local density and the properties of nearest neighbor. Thus, it is necessary to separate the effects of both environments.

In previous studies we have examined the effects of both large- and small-scale environments on the SFA and the morphological transformation of SDSS galaxies (Park et al. 2008; Park \& Choi 2009; Hwang et al. 2010a), and on the morphological transformation of GOODS galaxies (Hwang \& Park 2009). We found that the SFA of local galaxies is still strongly affected by the nearest neighbors differently depending on the morphology even at fixed large-scale background density (see Fig. 14 in Hwang et al. 2010a and Fig. 7 in Park \& Choi 2009). The morphological transformation of high- $z$ galaxies shows a similar trend (see Fig. 6 in Hwang \& Park 2009). Therefore, it is expected that the role of the large-scale environment in the SFA for high- $z$ galaxies to be weak. We tried to investigate the change in SFRs of GOODS galaxies depending on both large- and smallscale environments, and found that galaxies with early (late)type neighbors are not preferentially selected in high (low)density regions. This implies that there is no bias in our results introduced by the difference in the large-scale environment (see also Fig. 6 in Hwang \& Park 2009). However, we could not draw meaningful conclusions on the distinction of the effects of largeand small-scale environments on the SFA because of the small number statistics, which needs to be investigated with a more comprehensive data set of IR-detected galaxies in future studies. On the other hand, a detailed analysis focusing on the effects of large-scale environment on the SFA of high- $z$ galaxies can be found in other studies based on similar GOODS data (Elbaz et al. 2007; Popesso et al. 2011a,b).

\section{Conclusions}

Using the Herschel-selected galaxies in the GOODS fields and the IRAS plus AKARI-selected galaxies in the field of SDSS DR7, we studied the impact of galaxy-galaxy interactions on the FIR properties of galaxies and its evolution at $0<z<1.2$. Our main results follow.

1. We found that the SFRs and SSFRs of galaxies, on average, depend on the morphology of and the distance to the nearest neighbor galaxy for all redshifts, within $0<z<1.2$. When a late-type galaxy has a close neighbor galaxy, the SFR and SSFR increase as it approaches a late-type neighbor, which is supported by $\mathrm{K}-\mathrm{S}$ and MC tests with a significance level of $>99 \%$. However, the SFR and SSFR decrease or do not change much as it approaches an early-type neighbor. The bifurcations of SFRs and SSFRs depending on the neighbor's morphology are seen at $R_{n} \approx 0.5 r_{\text {vir,nei }}$, which is also supported by $\mathrm{K}-\mathrm{S}$ and $\mathrm{MC}$ tests with a significance level of $>98 \%$.

2. For the redshift range $0<z<1.2$, the SSFRs of latetype galaxies with late-type neighbors are increased by factors of about $1.8 \pm 0.7$ and $4.0 \pm 1.2$, respectively, for the cases of weakly $\left(R_{\mathrm{n}} \leq 0.5 r_{\text {vir,nei }}\right)$ and strongly interacting $\left(R_{\mathrm{n}} \leq 0.1 r_{\mathrm{vir}, \mathrm{nei}}\right)$ galaxies compared to those of noninteracting galaxies.

3. The dust temperature of both local and high-z late-type galaxies strongly interacting with late-type neighbors appears to be higher than that of non-interacting galaxies with a significance leve of 96-99\%. However, the dust temperature of local late-type galaxies strongly interacting with earlytype neighbors seems to be lower than or similar to that of non-interacting galaxies.

Our results suggest that galaxy-galaxy interactions and mergers have been strongly affecting the SFA and the dust properties of star-forming galaxies over at least 8 billion years.

Acknowledgements. We would like to thank the anonymous referee for constructive comments that helped us to improve the manuscript. H.S.H. and D.E. acknowledge the support of the Centre National d'Études Spatiales (CNES). D.E. acknowledges financial support from the French Agence Nationale de la Recherche (ANR) project "HUGE", ANR-09-BLAN-0224. V.C. would like to acknowledge partial support from the EU ToK grant 39965 and FP7-REGPOT 206469. PACS has been developed by a consortium of institutes led by MPE (Germany) and including UVIE (Austria); KU Leuven, CSL, IMEC (Belgium); CEA, LAM (France); MPIA (Germany); INAFIFSI/OAA/OAP/OAT, LENS, SISSA (Italy); IAC (Spain). This development has been supported by the funding agencies BMVIT (Austria), ESA-PRODEX (Belgium), CEA/CNES (France), DLR (Germany), ASI/INAF (Italy), and CICYT/MCYT (Spain). SPIRE has been developed by a consortium of institutes led by Cardiff University (UK) and including Univ. Lethbridge (Canada); NAOC (China); CEA, LAM (France); IFSI, Univ. Padua (Italy); IAC (Spain); SNSB (Sweden); Imperial College London, RAL, UCL-MSSL, UKATC, Univ. Sussex (UK); and Caltech, JPL, NHSC, Univ. Colorado (USA). This development has been supported by national funding agencies: CSA (Canada); NAOC (China); CEA, CNES, CNRS (France); ASI (Italy); MCINN (Spain); Stockholm Observatory (Sweden); STFC (UK); and NASA (USA). This research is based on observations with AKARI, a JAXA project with the participation of ESA. Funding for the SDSS and SDSS-II has been provided by the Alfred P. Sloan Foundation, the Participating Institutions, the National Science Foundation, the US Department of Energy, the National Aeronautics and Space Administration, the Japanese Monbukagakusho, the Max Planck Society, and the Higher Education Funding Council for England. The SDSS Web Site is http://www.sdss.org/. The SDSS is managed by the Astrophysical Research Consortium for the Participating Institutions. The Participating Institutions are the American Museum of Natural History, Astrophysical Institute Potsdam, University of Basel, Cambridge University, Case Western Reserve University, University of Chicago, Drexel University, Fermilab, the Institute for Advanced Study, the Japan Participation Group, Johns Hopkins University, the Joint Institute for Nuclear Astrophysics, the Kavli Institute for Particle Astrophysics and Cosmology, the Korean Scientist Group, the Chinese Academy of Sciences (LAMOST), Los Alamos National Laboratory, the Max-Planck-Institute for Astronomy (MPIA), the Max-Planck-Institute for Astrophysics (MPA), New Mexico State University, Ohio State University, University of Pittsburgh, University of Portsmouth, Princeton University, the United States Naval Observatory, and the University of Washington. This research has made use of the NASA/IPAC Extragalactic Database (NED) which is operated by the Jet Propulsion Laboratory, California Institute of Technology, under contract with the National Aeronautics and Space Administration.

\section{References}

Abazajian, K. N., Adelman-McCarthy, J. K., Agüeros, M. A., et al. 2009, ApJS, 182,543

Alonso, M. S., Tissera, P. B., Coldwell, G., \& Lambas, D. G. 2004, MNRAS, 352,1081 
Ann, H. B., Park, C., \& Choi, Y. 2008, MNRAS, 389, 86 Balestra, I., Mainieri, V., Popesso, P., et al. 2010, A\&A, 512, A12 Barger, A. J., Cowie, L. L., \& Wang, W.-H. 2008, ApJ, 689, 687 Barton, E. J., Geller, M. J., \& Kenyon, S. J. 2000, ApJ, 530, 660 Bergvall, N., Laurikainen, E., \& Aalto, S. 2003, A\&A, 405, 31 Blanton, M. R., \& Roweis, S. 2007, AJ, 133, 734

Bridge, C. R., Carlberg, R. G., \& Sullivan, M. 2010, ApJ, 709, 1067 Brinchmann, J., Charlot, S., White, S. D. M., et al. 2004, MNRAS, 351, 1151 Chanial, P., Flores, H., Guiderdoni, B., et al. 2007, A\&A, 462, 81 Chary, R., \& Elbaz, D. 2001, ApJ, 556, 562 Choi, Y., Park, C., \& Vogeley, M. S. 2007, ApJ, 658, 884

Choi, Y., Han, D., \& Kim, S. S. 2010, J. Korean Astron. Soc., 43, 191 Cohen, J. G., Hogg, D. W., Blandford, R., et al. 2000, ApJ, 538, 29 Condon, J. J., Condon, M. A., Gisler, G., \& Puschell, J. J. 1982, ApJ, 252, 102

Cooper, M. C., Aird, J. A., Coil, A. L., et al. 2011, ApJS, 193, 14

Cowie, L. L., Barger, A. J., Hu, E. M., Capak, P., \& Songaila, A. 2004, AJ, 127, 3137

Daddi, E., Dickinson, M., Morrison, G., et al. 2007, ApJ, 670, 156

Darg, D. W., Kaviraj, S., Lintott, C. J., et al. 2010, MNRAS, 401, 1552

De Propris, R., Liske, J., Driver, S. P., Allen, P. D., \& Cross, N. J. G. 2005, AJ, 130,1516

de Ravel, L., Le Fèvre, O., Tresse, L., et al. 2009, A\&A, 498, 379

Dickinson, M., Giavalisco, M., \& GOODS Team. 2003, in The Mass of Galaxies at Low and High Redshift, ed. R. Bender, \& A. Renzini, 324

Dressler, A. 1980, ApJ, 236, 351

Elbaz, D., Daddi, E., Le Borgne, D., et al. 2007, A\&A, 468, 33

Elbaz, D., Hwang, H. S., Magnelli, B., et al. 2010, A\&A, 518, L29

Elbaz, D., Dickinson, M., Hwang, H. S., et al. 2011, A\&A, 533, A119

Ellison, S. L., Patton, D. R., Simard, L., \& McConnachie, A. W. 2008, AJ, 135, 1877

Feruglio, C., Aussel, H., Le Floc'h, E., et al. 2010, ApJ, 721, 607

Fioc, M., \& Rocca-Volmerange, B. 1999, astro-ph/9912179

Gallazzi, A., Charlot, S., Brinchmann, J., White, S. D. M., \& Tremonti, C. A. 2005, MNRAS, 362, 41

Geller, M. J., Kenyon, S. J., Barton, E. J., Jarrett, T. H., \& Kewley, L. J. 2006, AJ, 132, 2243

Giavalisco, M., Ferguson, H. C., Koekemoer, A. M., et al. 2004, ApJ, 600, L93

Gómez, P. L., Nichol, R. C., Miller, C. J., et al. 2003, ApJ, 584, 210

Griffin, M. J., Abergel, A., Abreu, A., et al. 2010, A\&A, 518, L3

Grützbauch, R., Trinchieri, G., Rampazzo, R., et al. 2007, AJ, 133, 220

Hernández-Toledo, H. M., Avila-Reese, V., Conselice, C. J., \& Puerari, I. 2005, AJ, 129, 682

Huang, M.-L., \& Hwang, C.-Y. 2011, ApJ, 734, 99

Hwang, H. S., \& Park, C. 2009, ApJ, 700, 791

Hwang, H. S., Elbaz, D., Lee, J. C., et al. 2010a, A\&A, 522, A33

Hwang, H. S., Elbaz, D., Magdis, G., et al. 2010b, MNRAS, 409, 75

Isobe, T., Feigelson, E. D., Akritas, M. G., \& Babu, G. J. 1990, ApJ, 364, 104

Kartaltepe, J. S., Sanders, D. B., Scoville, N. Z., et al. 2007, ApJS, 172, 320

Kauffmann, G., Heckman, T. M., White, S. D. M., et al. 2003, MNRAS, 341, 33

Kawada, M., Baba, H., Barthel, P. D., et al. 2007, PASJ, 59, 389

Keel, W. C., Kennicutt, Jr., R. C., Hummel, E., \& van der Hulst, J. M. 1985, AJ, 90, 708

Kennicutt, Jr., R. C. 1998, ARA\&A, 36, 189

Kennicutt, Jr., R. C., Roettiger, K. A., Keel, W. C., van der Hulst, J. M., \& Hummel, E. 1987, AJ, 93, 1011

Klaas, U., Haas, M., Müller, S. A. H., et al. 2001, A\&A, 379, 823

Knapen, J. H., \& James, P. A. 2009, ApJ, 698, 1437

Kroupa, P. 2001, MNRAS, 322, 231

Kurk, J., Cimatti, A., Zamorani, G., et al. 2009, A\&A, 504, 331

Lambas, D. G., Tissera, P. B., Alonso, M. S., \& Coldwell, G. 2003, MNRAS, 346,1189

Larson, R. B., \& Tinsley, B. M. 1978, ApJ, 219, 46

Le Borgne, D., \& Rocca-Volmerange, B. 2002, A\&A, 386, 446

Le Fèvre, O., Vettolani, G., Paltani, S., et al. 2004, A\&A, 428, 1043

Lee, J. H., Lee, M. G., Park, C., \& Choi, Y.-Y. 2010, MNRAS, 403, 1930

Lewis, I., Balogh, M., De Propris, R., et al. 2002, MNRAS, 334, 673

Li, C., Kauffmann, G., Heckman, T. M., Jing, Y. P., \& White, S. D. M. 2008, MNRAS, 385, 1903

Lin, L., Koo, D. C., Weiner, B. J., et al. 2007, ApJ, 660, L51
Lutz, D., Spoon, H. W. W., Rigopoulou, D., Moorwood, A. F. M., \& Genzel, R. 1998, ApJ, 505, L103

Magdis, G. E., Elbaz, D., Hwang, H. S., et al. 2010a, MNRAS, 409, 22

Magdis, G. E., Elbaz, D., Hwang, H. S., et al. 2010b, ApJ, 720, L185

Magdis, G. E., Rigopoulou, D., Huang, J., \& Fazio, G. G. 2010c, MNRAS, 401, 1521

Magdis, G. E., Elbaz, D., Dickinson, M., et al. 2011, A\&A, 534, A15

Mignoli, M., Cimatti, A., Zamorani, G., et al. 2005, A\&A, 437, 883

Moshir, M., Kopman, G., \& Conrow, T. A. O. 1992, IRAS Faint Source Survey, Explanatory supplement version 2

Murakami, H., Baba, H., Barthel, P., et al. 2007, PASJ, 59, 369

Nikolic, B., Cullen, H., \& Alexander, P. 2004, MNRAS, 355, 874

Park, C., \& Choi, Y. 2005, ApJ, 635, L29

Park, C., \& Choi, Y. 2009, ApJ, 691, 1828

Park, C., \& Hwang, H. S. 2009, ApJ, 699, 1595

Park, C., Choi, Y., Vogeley, M. S., Gott, J. R. I., \& Blanton, M. R. 2007, ApJ, 658, 898

Park, C., Gott, J. R. I., \& Choi, Y. 2008, ApJ, 674, 784

Patel, S. G., Holden, B. P., Kelson, D. D., Illingworth, G. D., \& Franx, M. 2009, ApJ, 705, L67

Patton, D. R., Pritchet, C. J., Yee, H. K. C., Ellingson, E., \& Carlberg, R. G. 1997, ApJ, 475, 29

Patton, D. R., Grant, J. K., Simard, L., et al. 2005, AJ, 130, 2043

Patton, D. R., Ellison, S. L., Simard, L., McConnachie, A. W., \& Mendel, J. T. 2011, MNRAS, 412, 591

Peebles, P. J. E. 1993, Principles of physical cosmology, ed. P. J. E. Peebles

Perez, J., Tissera, P., Padilla, N., Alonso, M. S., \& Lambas, D. G. 2009, MNRAS, 399,1157

Pilbratt, G. L., Riedinger, J. R., Passvogel, T., et al. 2010, A\&A, 518, L1

Poglitsch, A., Waelkens, C., Geis, N., et al. 2010, A\&A, 518, L2

Popesso, P., Dickinson, M., Nonino, M., et al. 2009, A\&A, 494, 443

Popesso, P., Rodighiero, G., Saintonge, A., et al. 2011a, A\&A, 532, A145

Popesso, P., et al. 2011b, A\&A, in press, DOI: 10.1051/0004-6361/201117973

Ravikumar, C. D., Puech, M., Flores, H., et al. 2007, A\&A, 465, 1099

Reddy, N. A., Steidel, C. C., Erb, D. K., Shapley, A. E., \& Pettini, M. 2006, ApJ, 653, 1004

Retzlaff, J., Rosati, P., Dickinson, M., et al. 2010, A\&A, 511, A50+

Rigopoulou, D., Spoon, H. W. W., Genzel, R., et al. 1999, AJ, 118, 2625

Salpeter, E. E. 1955, ApJ, 121, 161

Sanders, D. B., Mazzarella, J. M., Kim, D., Surace, J. A., \& Soifer, B. T. 2003, AJ, 126, 1607

Schlegel, D. J., Finkbeiner, D. P., \& Davis, M. 1998, ApJ, 500, 525

Silverman, J. D., Mainieri, V., Salvato, M., et al. 2010, ApJS, 191, 124

Smith, B. J., Struck, C., Hancock, M., et al. 2007, AJ, 133, 791

Struck, C. 2006, Galaxy Collisions - Dawn of a New Era, ed. Mason, J. W. (Springer Verlag), 115

Szokoly, G. P., Bergeron, J., Hasinger, G., et al. 2004, ApJS, 155, 271

Tegmark, M., Blanton, M. R., Strauss, M. A., et al. 2004, ApJ, 606, 702

Telesco, C. M., Wolstencroft, R. D., \& Done, C. 1988, ApJ, 329, 174

Toomre, A. 1977, in Evolution of Galaxies and Stellar Populations, ed. B. M. Tinsley, \& R. B. Larson, 401

Vanzella, E., Cristiani, S., Dickinson, M., et al. 2005, A\&A, 434, 53

Vanzella, E., Cristiani, S., Dickinson, M., et al. 2006, A\&A, 454, 423

Vanzella, E., Cristiani, S., Dickinson, M., et al. 2008, A\&A, 478, 83

Wang, W.-H., Cowie, L. L., Barger, A. J., Keenan, R. C., \& Ting, H.-C. 2010a, ApJS, 187, 251

Wang, Y., Park, C., Hwang, H. S., \& Chen, X. 2010b, ApJ, 718, 762

Wirth, G. D., Willmer, C. N. A., Amico, P., et al. 2004, AJ, 127, 3121

Wolf, C., Meisenheimer, K., Rix, H., et al. 2003, A\&A, 401, 73

Wong, K. C., Blanton, M. R., Burles, S. M., et al. 2011, ApJ, 728, 119

Woods, D. F., \& Geller, M. J. 2007, AJ, 134, 527

Woods, D. F., Geller, M. J., Kurtz, M. J., et al. 2010, AJ, 139, 1857

Xia, L., Malhotra, S., Rhoads, J., et al. 2011, AJ, 141, 64

Xilouris, E. M., Georgakakis, A. E., Misiriotis, A., \& Charmandaris, V. 2004 MNRAS, 355, 57

Xu, C. K., Domingue, D., Cheng, Y., et al. 2010, ApJ, 713, 330

Yee, H. K. C., \& Ellingson, E. 1995, ApJ, 445, 37

York, D. G., Adelman, J., Anderson, Jr., J. E., et al. 2000, AJ, 120, 1579

Zepf, S. E., \& Koo, D. C. 1989, ApJ, 337, 34 